\title{
Validation of joint angle measurements: Comparison of a novel low cost marker-less system with an industry standard marker-based system.
}

Shayan Bahadori ${ }^{1}$ (corresponding author)

Philip Davenport ${ }^{2}$

Tikki Immins ${ }^{1}$

Thomas W. Wainwright ${ }^{1}$

Email: sbahadori@bournemouth.ac.uk.Telephone: +44 (0)1202961647

Email: pdavenport@bournemouth.ac.uk.Telephone: +44 (0)1202961994

Email: timmins@bournemouth.ac.uk.Telephone: +44 (0)1202962727

Email: twainwright@bournemouth.ac.uk.Telephone: +44 (0)1202961656

${ }^{1}$ Orthopaedic Research Institute, Bournemouth University, 6th Floor Executive Business Centre, 89 Holdenhurst Road, Bournemouth, BH8 8EB, UK.

${ }^{2}$ Faculty of Science and Technology, Bournemouth University, Poole House, Talbot Campus, Poole, BH12 5BB, UK.

\section{Corresponding Author:}

Shayan Bahadori, Orthopaedic Research Institute, Bournemouth University, 6th Floor Executive Business Centre, 89 Holdenhurst Road, Bournemouth, BH8 8EB, UK.

Email: sbahadori@bournemouth.ac.uk 


\begin{abstract}
Human motion tracking is widely used for assessment of movement dysfunction in orthopaedic patients. Currently, most clinical motion analysis centres use marker based three-dimensional (3D) systems as they are deemed to be the most accurate method. However, due to space, costs and logistics they are not available in many clinical settings. This study compared joint angles measured in functional tests using the novel low-cost Microsoft Kinect Perfect Phorm system with the established marker based Nexus VICON system. When measuring right and left knee flexion, the average difference between the VICON and Kinect Perfect Phorm measurement was 13.2\%, with a SD of 19.6. Both overestimation and underestimation of the joint angle was recorded in different participants. Although the average percentage difference during hip abduction tests was lower at $-3.9 \%$, the range of error was far greater $(S D=75)$. From this, it can be concluded that the level of accuracy presented in the new low cost Kinect Perfect Phorm system is not yet suitable for clinical assessments. However, for general tests of performance, and for tracking cases where absolute accuracy is less critical, future versions of this software may have a place.
\end{abstract}

\title{
Keywords
}

Kinect, Nexus VICON, motion capture, movement analysis, system performance. 


\section{Introduction}

3D motion capture with marker-based tracking systems are recognised as the current gold standard in human movement evaluation [1]. Such systems are used in the clinical evaluation and assessment of a range of conditions, including planning for multi-level surgery in cerebral palsy, studies into the suitability of orthotic or prosthetic interventions, and research into many aspects of movement [2]. To achieve results of sufficient accuracy for clinical use, highly skilled and well-trained staff are required to calibrate and run the system. Such equipment is available from a range of commercial suppliers, but systems are expensive to set up, require a large testing space and take time to process and obtain results. This can limit the settings in which these measurements can take place, with the effect that many clinical environments are unable to make reliable quantitative measurements of movement.

In recent years, motion analysis technology has proliferated in the entertainment sector. Devices such as the Nintendo Wii or Microsoft Kinect have been used to track the movement of game players, and the technology has been adapted for use in medical environments [3, 4]. This is attractive for a number of reasons, including the low price of components and the portability and ease of use of the devices. This approach could therefore be used in a wider range of care settings, and provide a convenient method of providing these measurements.

Recently a system called Perfect Phorm (Seebook Technology LLC, Dallas, USA. http://perfectphorm.com/) has been developed that purports to reliably estimate body kinematics using a marker-less system that uses Microsoft Kinect in combination with custom software. The aim of this study was to evaluate the Kinect Perfect Phorm system compared to the current gold-standard Nexus-VICON system [5,6]. A series of specified functional tests are performed in unimpaired participants, with the aim to examine the relative accuracy of the Kinect Perfect 
Phorm system and the correlation between the two systems.

\section{Methodology}

Ten unimpaired volunteers (five male, five female; 23 - 61 years; $164 \pm 11 \mathrm{~cm}$ height) were recruited in May 2017 for this study. Participants were required to perform simple movements without difficulty, and were excluded if they reported recent significant joint or bone injury, or any muscle or neurological condition that had the potential to affect movement. Each participant’s age, weight and height were recorded.

Participants were fitted with 16 retroreflective markers in the positions specified by the Plug-inGait (PiG) lower limb marker set [7]. Measurements of knee and ankle width bilaterally were recorded to facilitate processing of the biomechanical model.

Participants were asked to make three movements: standing, a squat action and a hip abduction action. The standing action was used to calibrate the labelling model in use by the biomechanical model. The squat action consisted of a recording of comfortable standing for approximately five seconds, moving into a squat position of knee and ankle flexion, also held for five seconds, then a return to comfortable standing for a final five seconds. The hip abduction task also started with five seconds comfortable standing, followed by an action where the left hip was abducted and held steady for five seconds, and then returned to comfortable standing for five seconds. Each participant was allowed practice for the required actions, and was able to self-select the degree of motion used. Examples of the actions are shown in figure 1. The squat and hip abduction tasks were each repeated three times for each participant. 
Figure 1 - Example of actions. A) standing. B) hip abduction. C) Squat. D) active marker from Kinect Perfect Phorm system is shown on the joints. E) VICON skeleton model of the participant during the standing action.

The two measurement systems were used simultaneously to measure changes in joint angle. The gold standard measurement was a 10 camera VICON system that formed part of a GRAIL gait measurement system. The system ran using VICON 2.3 software, the lower-limb plug-in-gait version 3.1.1 model and was calibrated on the day of testing using an active wand by an experienced technician. Each task was evaluated with the participant standing on a (deactivated) split-belt treadmill in the centre of the calibrated capture volume. Handrails were available for balance if required.

The second measurement system was a Kinect Perfect Phorm device consisting of a laptop and Microsoft Kinect camera and Perfect Phorm GM software (PPGM), version 1.0.0.320. This was placed approximately three meters away from the participant such that a clear view of the participant's whole body was in frame. The arrangement of both systems is shown in figure 2: the Kinect Perfect Phorm system was positioned in the participant's coronal plane.

Figure 2 - Laboratory layout. A) Schematic of the locations for VICON cameras and Kinect Perfect Phorm Kinect system. B) Treadmill location in the centre of the gait laboratory. C) Microsoft Kinect location; facing the treadmill. D) Microsoft Kinect is mounted onto a tripod so it can be adjusted to the angle and height of the participant for a full body view.

The collection of data from each participant was synchronised manually between each system. Average values for left and right knee flexion (for the squat task) and left hip abduction (for the hip abduction task) were obtained from each system as the average across the steady state, and the relative change between the standing and task sections used as the basis of comparisons of 
system performance.

The study protocol was reviewed and approved by the Bournemouth University research ethics committee (Reference 14708), and each volunteer provided their written informed consent for participation. Participants were recruited from the local student population and faculty.

\section{Data Analysis}

An example of the collected movement data for a participant completing the squat and hip extension tasks is shown in figure 3. For each participant, the steady state joint angle during rest and the action at each knee (for the squat task) and the left hip (in the hip abduction task) was obtained from the processed VICON model and from the PPGM software. This was recorded as the mean over an approximately two second window in each trial. The difference between the rest and action joint angles was calculated, and the difference between the VICON and Kinect Perfect Phorm data expressed as a percentage of the VICON measurement. This acted to normalise the evaluation of the difference in estimate between systems as each participant was free to select the degree of movement in each action. The mean and SD of percentage differences for each action were calculated.

The distributions of results from the Kinect Perfect Phorm and VICON systems were assessed using the Kolmogorov-Smirnoff test. As normality could not be assumed, the non-parametric test, the Spearman's Rank Correlation Coefficient was calculated to measure the strength of the association between the results from the two systems.

Figure 3 - Hip abduction task and left and right knee flexion task position data. 


\section{Results}

All ten participants were able to complete all tasks requested of them. During analysis, marker occlusion meant that one set of VICON data (Participant 3, Knee squat attempt 3) was not complete, and so was removed from analysis. One participant’s Kinect Perfect Phorm joint angles were not successfully recorded during the hip abduction task (Participant 5), and were also excluded. Therefore, results were available from 10 participants (29 total trials) for the knee squat task, and 9 participants (27 total trials) for the hip abduction task.

Figure 4 - The average percentage difference in joint angle (mean +/- 1SD) for the left and right knee (during the squat task) and left hip (during the hip abduction task).

Table 1 - Summary of error (mean percentage difference and standard deviation) and correlations (Spearmans Rho).

When Spearman's rank correlation was calculated, the left knee flexion test showed high positive correlation between the VICON and Kinect Perfect Phorm measurement. A positive correlation was also present in the right knee flexion. A poor correlation was calculated for hip measurements changes. 


\section{Discussion}

The Kinect Perfect Phorm system was capable of recording changes in joint angle during knee and hip movement tasks which broadly corresponded to those recorded by the gold standard VICON system. However, although the overall pattern of motion was obtained, there remained substantial differences in the recorded joint angle.

Previous work in the field of movement analysis has suggested that a difference of 3-5\% could be considered clinically relevant $[8,9]$. When measuring right and left knee flexion, the average difference between the VICON and Kinect Perfect Phorm measurement was $13.2 \%$, with a SD of 19.6. Both overestimation and underestimation of the joint angle was recorded in different participants. Although the average percentage difference during hip abduction tests was lower at $-3.9 \%$, the range of errors was far greater $(\mathrm{SD}=75)$.

The relatively better accuracy of the Kinect Perfect Phorm system during the knee flexion test was somewhat surprising given that the main axis of joint flexion was in the sagittal plane and the Kinect camera was observing in the coronal plane (Figure 4). As the changes in the sagittal plane must be evaluated using the depth information rather than the in-plane movement which could be observed via the image, it was thought that these movements would be more difficult to evaluate accurately. In practice, the hip abduction movement, which was substantially in the coronal plane, demonstrated a much greater range of errors. It should be noted that these results are consistent with recently published work in dynamic movements [6].

A small difference in VICON measures of knee motion between left and right knee was observed. As between knee asymmetry is considered unlikely, this could be attributed to minor crosstalk between the actual knee flexion axis and that modelled by the Plug-in-Gait model as a 
result of imperfect reflective marker placement [11]. Similarly, it is possible that as the Kinect camera was placed closely but not perfectly parallel to the participant's frontal plane, that some error in the calculation of joint angles could arise. However, the instruction manual for the Kinect Perfect Phorm system does not place specific restrictions on the placement of the camera.

The low correlation between VICON and Kinect Perfect Phorm measurements is of concern when considering a potential clinical application for this tool. A consistent inaccuracy could be accounted for in the interpretation of results; however the poor correlation between the gold standard and the Kinect Perfect Phorm measurements means that clinicians cannot place much confidence in the quality of the device output.

One limitation of the study is that a precise evaluation of the repeatability of each measurement system was not possible. As participants were not asked to replicate motions and position exactly, a measure of absolute accuracy was not obtained. Instead, the VICON system was treated as a gold standard, and the change in joint angles measured by the Kinect Perfect Phorm system was compared to this, rather than attempting a repeatability analysis. In practice, participants were fairly consistent in their self-selected movement range as measured by the VICON system.

A limitation of the Kinect Perfect Phorm system can be seen in the example left knee flexion graph (Figure 3). The fluctuation visible on the Kinect Perfect Phorm knee flexion trace in the flexed position could be observed in several participants' measurements, and suggests that the Kinect Perfect Phorm data can suffer from poor tracking in some situations. The PPGM software also lacks filtering capability. Given that it is not reasonable for most clinicians to implement this independently, it should be a consideration for the future development of this system. 
Despite poor performance on the hip abduction measurement, much stronger performance was evident on the knee flexion movements. These demonstrated a lower standard deviation between participants in terms of accuracy of measurement, and a much greater positive correlation between the VICON and Kinect Perfect Phorm measurements. This suggests that for some forms of movement in some joints, the PPGM software may be capable of performing adequate assessments of movement. Previous literature has suggested that a 5 degree inaccuracy would be insufficient for use in clinical assessments [10, 12]. In the tests completed in this study, a 5 degree error, expressed as a percentage of the overall change in joint angle, would represent an error of between 3.4\% (for the greatest change in joint angle) and 13.2\% (for the smallest change in joint angle).

\section{Conclusions}

It can be concluded that the level of accuracy presented in the new low cost Kinect Perfect Phorm system is not yet suitable for clinical assessments. However, for general tests of performance, and for tracking cases where absolute accuracy is less critical, future versions of this software may have a place. 


\section{References}

[1] Zhou H, Hu H. Human motion tracking for rehabilitation - a survey. Biomed Signal Process Control. 2008;3:1-18.

[2] McGinley J, Baker R, Wolfe R, et al. The reliability of three-dimensional kinematic gait measurements: A systematic review. Gait Posture. 2009;29:360-369.

[3] Chang U, Chen S, Huang J. A Kinect-based system for physical rehabilitation: A pilot study for young adults with motor disabilities. Res dev Disabil. 2011;32:2566-2570.

[4] Fernandez-Baena A, Susin A, Ligadas X. Biomechanical Validation of Upper-body and Lower-body Joint Movements of Kinect Motion Capture Data for Rehabilitation Treatments. In Fourth Int. Conference on Intelligent Networking and Collaborative Systems (INCoS’12), proceedings, 656-661, Bucharest, Romania, September 2012.

[5] Bonnechere B, Jansen B, Moiseev F, et al. Validity and reliability of the Kinect within functional assessment activities - Comparison with standard stereophotogrammetry. Gait \& Posture. 2014;39:593-598.

[6] Pfister A, West A, Bonner S, et al. Comparative abilities of Microsoft Kinect and Vicon 3D motion capture for gain analysis. Medical Engineering \&Technology. 2014; 38:274-280.

[7] Vicon.com [Internet]; United Kingdom. 2017 [Feb 20 ${ }^{\text {th }}$. Available from: https://docs.vicon.com/display/Nexus25/Lower+body+modeling+with+Plug-in+Gait.

[8] Saito H, Watanabe T, Arifin A. Ankle and Knee Joint Angle Measurements during Gait with Wearable Sensor System for Rehabilitation. World Congress on Medical Physics and Biomedical Engineering, IFMBE proceedings, September, 2009, Munich, Germany. 
[9] Watanabe T, Saito H, Koike E, et al. A Preliminary Test of Measurement of Joint Angles and Stride Length with Wireless Inertial Sensors for Wearable Gait Evaluation System. Comput Intel Neurosc. 2011;2011:975193.

[10] Luttgens K, Hamilton N. Kinesiology: Scientific Basis of Human Motion. Dubuque (IA): Brown and Benchmark; 2011.

[11] Schache A, Baker R, Lamoreux LW. Defining the knee joint flexion-extension axis for purposes of quantitative gait analysis: An evaluation of methods. Gait \& Posture. 2006; 24(1) 100-109.

[12] McGinley J, Baker R, Wolfe R, et al. The reliability of three-dimensional kinetic gait measurements: A systematic review. Gait \& Posture. 2009; 29(3) 360-369. 